\title{
Awareness of Anti-Sialogogues Among Dental Students
}

\author{
P. Deeksheetha and Dhanraj Ganapathy \\ ${ }^{1}$ Saveetha Dental College and Hospitals, Saveetha Institute of Medical \\ and Technical Sciences, Saveetha University, Chennai - 600077 \\ Professor and Head of the Department, Department of Prosthodontics, \\ Saveetha Dental College and Hospitals, Saveetha Institute of Medical \\ and Technical Sciences, Saveetha University, Chennai - 600077, India
}

\section{ABSTRACT}

The aim of this research is to assess the awareness of anti-sialogogues among dental students. Antisialogogues are substances that decrease the production of saliva, and their effect is opposite to that of sialogogues. Sialogogues are agents or drugs which increase the salivation during xerostomia. Anti-Sialogogues may be natural or synthetic. The most common adverse effect of root canal treatments, extractions and jaw surgeries. Antisialogogues are contraindicated in cases of Sjogren's syndrome, and in patients undergoing radiation therapy. This was an epidemiological survey conducted on 100 CRI students. The questionnaire was distributed via an online survey tool. The questionnaire contained 10 open ended questions regarding the knowledge and awareness of antisialogogues. The responses were collected and tabulated in the excel sheets, following which they were exported to SPSS software for statistical analysis. The CRIs possessed a moderate amount of awareness on antisialogogues. $73 \%$ of the students were aware of the function of the antisialogogues. 85\% preferred the use of antisialogogues in cases of patients with hypersalivation. $37 \%$ of the students agreed that nausea is one of the side effects of antisialogogues, 79\% of the students were aware that antisialogogues are contraindicated in patients with sjogren's syndrome and in patients undergoing radiation therapy.

KEY WORDS: ANTI-SIALOGOGUES; ATROPINE; HYPERSALIVATION; SALIVA.

\section{INTRODUCTION}

Saliva plays an important role in maintaining the homeostasis in the oral cavity. Saliva is predominantly secreted by the major and minor salivary glands. At rest without exogenous or pharmacological simulation there is a small continuous salivary flow and an unstimulated

\section{ARTICLE INFORMATION}

*Corresponding Author:dhanraj@saveetha.com

Received 11th June 2020 Accepted after revision 10th August 2020 Print ISSN: 0974-6455 Online ISSN: 2321-4007 CODEN: BBRCBA

Thomson Reuters ISI Web of Science Clarivate Analytics USA and Crossref Indexed Journal

\section{Clarivate}

NAAS Journal Score 2020 (4.31) SJIF: 2020 (7.728)

A Society of Science and Nature Publication,

Bhopal India 2020. All rights reserved.

Online Contents Available at: http//www.bbrc.in/

Doi: http://dx.doi.org/10.21786/bbrc/13.7/29 secretion of salivary flow present in tissues. (Christensen, 1986) This flow of saliva at rest is about $0.4-0.5 \mathrm{ml} / \mathrm{min}$ in healthy subjects. Stimulated saliva is produced in response to a mechanical, gustatory, olfactory, or pharmacological stimulus, contributing to around $40-50 \%$ of daily salivary production. The Salivary Flow (SF) index is a parameter allowing stimulated and unstimulated saliva flow to be classified as normal, low or very low (hyposalivation). In adults, normal total stimulated SF ranges $1-3 \mathrm{~mL} /$ minute, low ranges $0.7-1.0 \mathrm{~mL} /$ minute, while hypo salivation is characterised by a stimulated $\mathrm{SF}<0.7 \mathrm{~mL} /$ minute. (Chainani-Wu et al., 2006).

In hypersalivation, the salivary glands produce more saliva than usual.Hypersalivation may be temporary or chronic depending on the cause. Constant hypersalivation

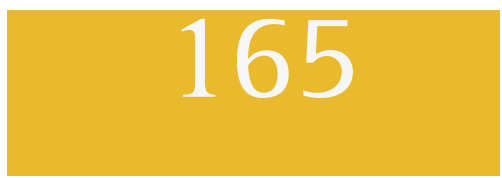


(sialorrhea) often relates back to an underlying condition that affects muscle control. Temporary hypersalivation is usually caused by: Cavities, infection, gastroesophageal reflux, pregnancy, certain tranquilizers and anticonvulsant drugs, exposure to toxins, such as mercury. (Mccubbin et al., 1979). In these cases, hypersalivation typically goes away after treating the underlying condition.Women who are pregnant typically see a decrease in symptoms after childbirth. Constant hypersalivation is usually caused by chronic health conditions that affect muscle control, when there is an impaired muscle control, it can affect the ability to swallow, leading to saliva buildup. This can result from:malocclusion, enlarged tongue, intellectual disability, cerebral palsy, facial nerve palsy, Parkinson's diseases, amyotrophic lateral sclerosis (ALS), stroke. When the cause is chronic, symptom management is key. ('Hypersalivation (Ptyalism)', 2012).

Commonly used Anti-sialogogues are Glycopyrrolate (Cuvposa), Atropine, Scopalmine, Botulinum Toxin The medications block the nerve impulses to the salivary glands so that they produce less saliva. (Murai et al., 1996). However, Anti-sialogogues can have some severe side effects, including: dry mouth, constipation, trouble urinating, blurred vision, hyperactivity, irritability. Scopolamine (Hyoscine) is given as a skin patch that's placed behind the ear. It works by blocking nerve impulses to the salivary glands. Its side effects include dizziness, rapid heartbeat, blurred vision, drowsiness.

Injections such as Botulinum toxin (Botox) injections if your hypersalivation is constant. The drug into one or more of the major salivary glands. (Kuijpers et al., 2010). The toxin paralyzes the nerves and muscles in the area, preventing the glands from producing salivaIn severe cases, this condition can be treated with surgery on the major salivary glands. The glands are removed completely or relocated so that the saliva is released in the back of the mouth where it can be easily swallowed. Excess salivation can hinder in delivering proper dental treatment by the dentist to their patients. (Saleh and Elazzazi, 2014). Moreover hypersalivation can cause improper bonding of dental cements to the tooth structure.

Previously our department has published extensive research on various aspects of prosthetic dentistry ('Evaluation of Corrosive Behavior of Four Nickelchromium Alloys in Artificial Saliva by Cyclic Polarization Test:An in vitro Study', 2017; Ganapathy, Kannan and Venugopalan, 2017; Jain, 2017a, 2017b; Ranganathan, Ganapathy and Jain, 2017; Ariga et al., 2018; Gupta, Ariga and Deogade, 2018; Anbu et al., 2019; Ashok and Ganapathy, 2019; Duraisamy et al., 2019; Varghese, Ramesh and Veeraiyan, 2019), this vast research experience has inspired us to research about the awareness of anti sialogogues.

\section{MATERIALS AND METHODS}

This was an epidemiological questionnaire based study that assessed the awareness of anti sialogogues among undergraduate students. The ethical board clearance was obtained from the IERB of Saveetha dental college and hospitals.

Sampling: The entire population of 100 CRIs were involved. There were no chances of sampling bias as the entire sample was involved. Good external validity for the present study, as the full population cluster was involved and the results obtained can be generalisable within the state and country in which the students have obtained similar training.

Data collection: The data collection was done via Google forms. The questionnaire contained questions about the awareness, practice and knowledge of antisialogogues. The content validity was assessed by average congruency percentage and cronbach's alpha was used to assess the reliability of the questionnaire. Google forms were used to circulate the questionnaire among the students. The data obtained was collected and tabulated using excel sheets the data was transferred to SPSS software by IBM for statistical analysis.

Statistics: The data was transferred to SPSS software by IBM, version 25 for windows OS. In which the output variables were defined.

\section{RESULTS AND DISCUSSION}

A total of 100 CRI students participated in this questionnaire study.

Figure 1: Awareness of anti sialogogues

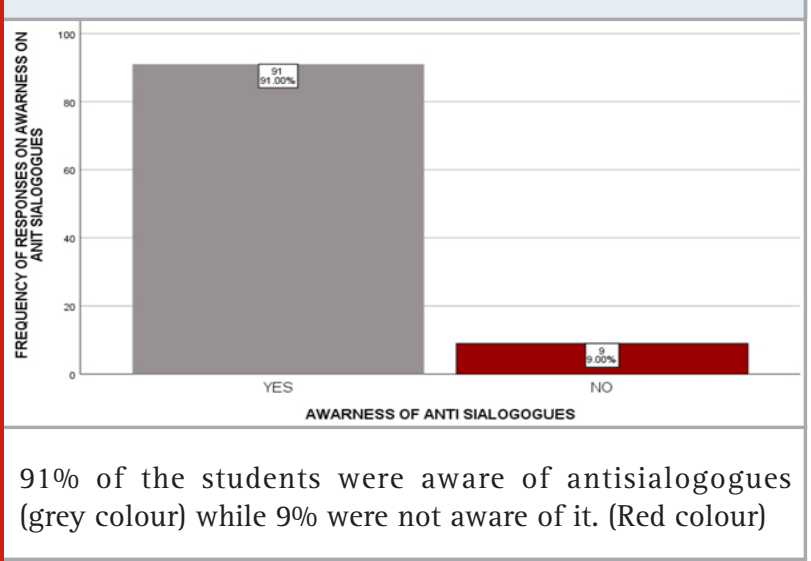

In a similar survey conducted by Revathy et al, 87\% of the students were aware of antisialogogues. 83\% were aware that antisialogogues reduced salivary flow the $20 \%$ said it as some oral medication drug and the remaining 7\% were not sure about the function of the anti sialogogues. 98\% do not use anti sialogogues in their daily practice. $31 \%$ of the participants agreed that the usage of anti sialogogues decreased chair side time. 27\% of the participants reported that they would prefer the use of anti sialogogues in patients with hypersalivation. (Revathy, 2017). These results were similar to the results obtained by the present study. Excess salivation can hinder successful jaw surgeries to bonding of light 
cured composite restorations to tooth structure. Anti sialogogues are substances which can reduce the salivary flow, and their action is opposite to that of sialogogues which are used in patients with xerostomia. (Singh et al., 2020).

Figure 2: Function of anti sialogogues

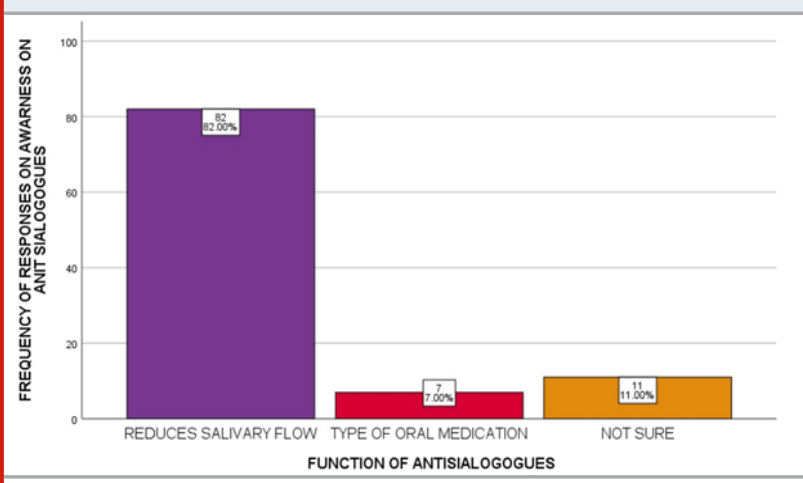

$82 \%$ of the study population were aware that the antisialogogues reduce salivary flow (violet colour), while $7 \%$ of the study population thought it was a type of oral medication (red colour) and the rest $11 \%$ of the study population was not sure of the role of anti sialogogues (yellow colour)

Figure 3: Clinical usage of anti sialogogues

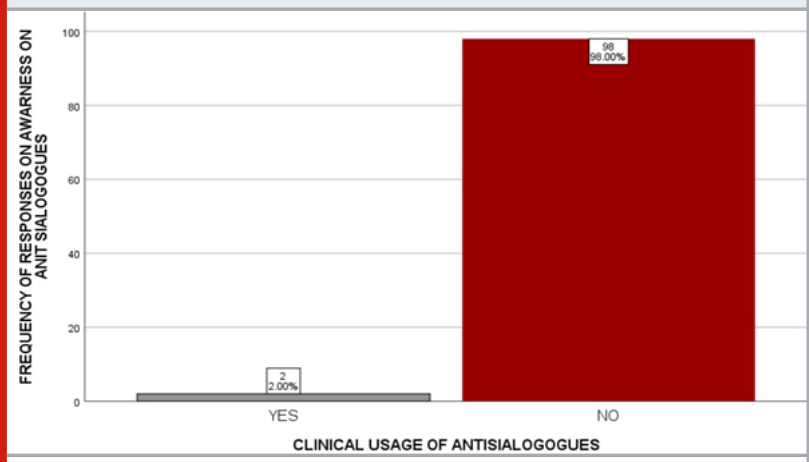

$98 \%$ of the students did not or have not used antisialogogues in their daily dental practice (red colour), only $2 \%$ of the study population have used antisialogogues in their daily practice (grey colour).

Other methods of isolation of the oral cavity include Rubber dam, introduced by SC Barnum, suction tips, svedopters, which are more commonly used to anti sialogogues. (Edwards, 1998) Commonly used anti sialogogue drugs are Atropine, Scopolamine, and hyoscyamine. Normally $0.6 \mathrm{mg}$ of atropine is given. Other lesser known anti sialogogues are Glycopyrrolate, Methantheline, Propantheline. (Leone, Georgievski and Koklanis, 2010). These anti sialogogues function from 20- 300 minutes after the injection which is dose dependent. Anti sialogogues are administered either via an intramuscular injection or as an oral tablet an hour prior to the dental procedure in patients with hypersalivation. (Gurney, 1967). Anti sialogogues like all medicines have their own set of side effects which are mostly nausea, constipation, tachycardia with an initial bradycardia, dry eyes, hyperthermia, headache, irritation, pupillary dilation. An initial bradycardia is more pronounced in the usage of IM propantheline.

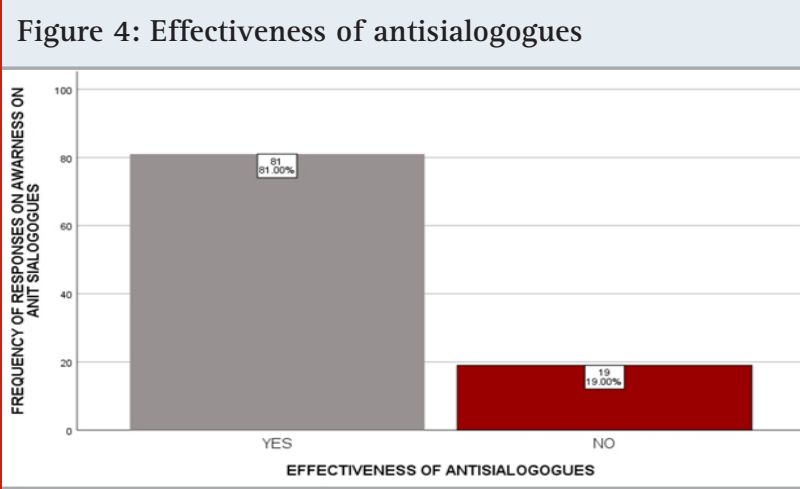

$81 \%$ of the study population felt that anti sialogogues are effective in clinical practice (grey colour) while the rest $19 \%$ felt that anti sialogogues are not effective in daily practice.

Figure 5: Reduction of chair side time

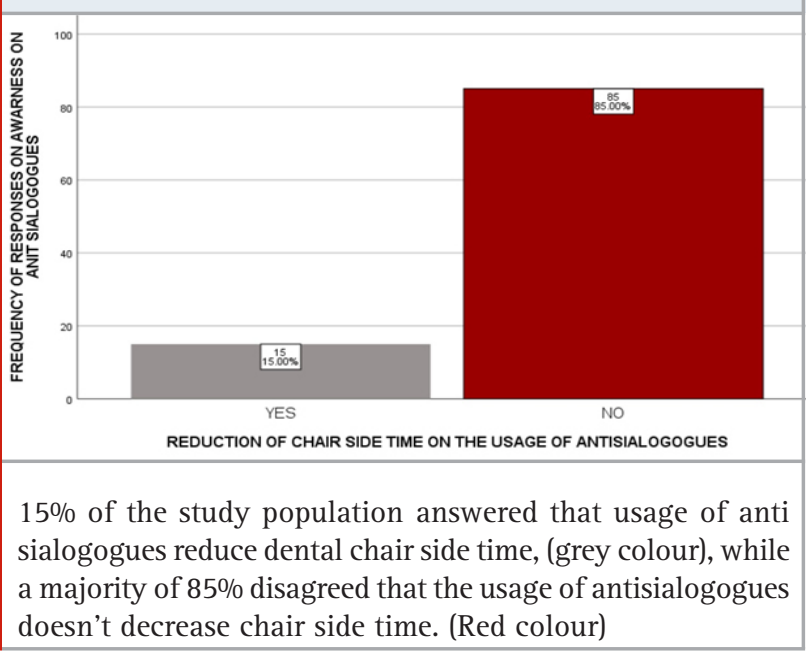

Figure 6: Usage of anti sialogogues during hypersalivation

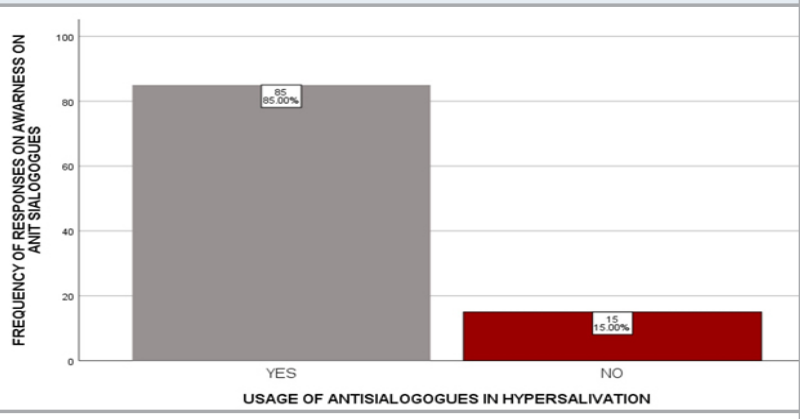

$85 \%$ of the study population agreed that they would prefer the usage of anti sialogogues in patients with hypersalivation (grey colour), while $15 \%$ of the study population did not prefer the usage of antisialogogues in patients with hypersalivation (red colour). 
Figure 7: Adverse effects of anti sialogogues

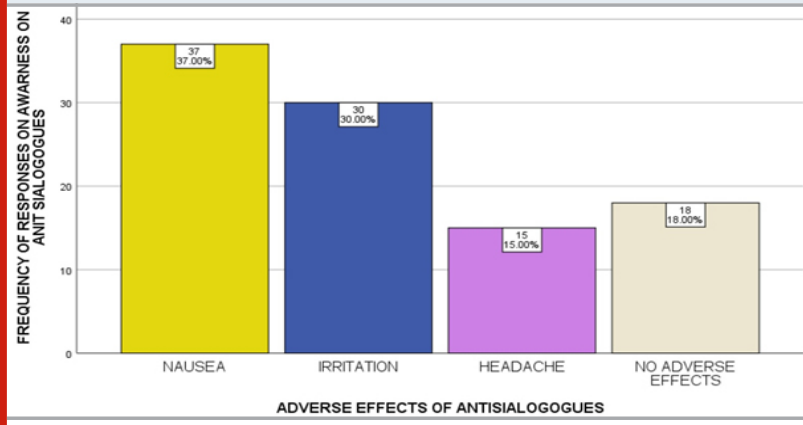

$37 \%$ of the students felt that nausea is an adverse effect of antisialogogue usage (yellow colour), 30\% felt that headache was an adverse effect (blue colour), while 15\% felt that headache was a side effect of antisialogogue usage (purple colour), while $18 \%$ felt that there are no adverse effects of anti sialogogues (cream colour).

Figure 8: Nature of Anti sialogogues

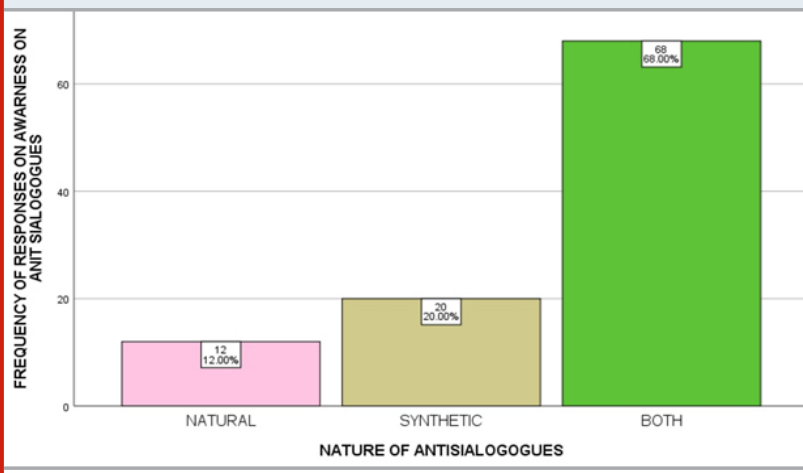

$12 \%$ of the study population thought antisialogogues were natural, (pink colour), while 20\% thought it was synthetic (mustard colour), 68\% of the study population answered correctly that anti sialogogues were both natural and synthetic (green colour).

Figure 9: Excess usage of anti sialogogues causes xerostomia

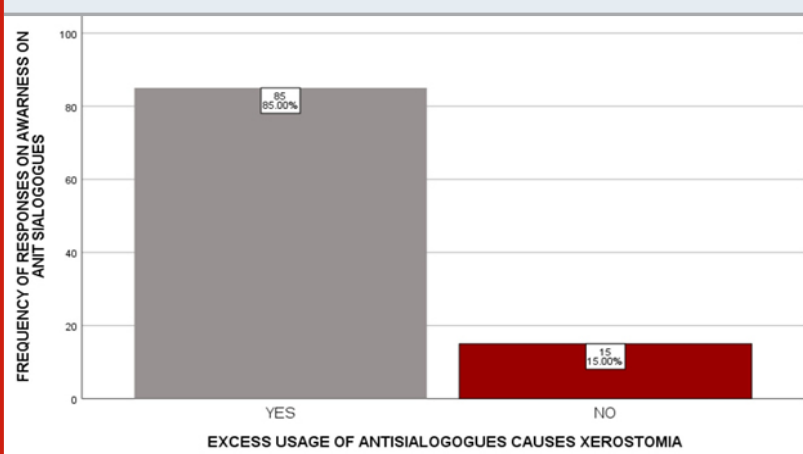

$85 \%$ of the study population were aware that the excess usage of antisialogogues can cause xerostomia (grey colour), while $15 \%$ of the study population were not aware that the excess usage of nati sialogogues causes xerostomia. (Red colour).
Figure 10: Contraindications of anti sialogogues

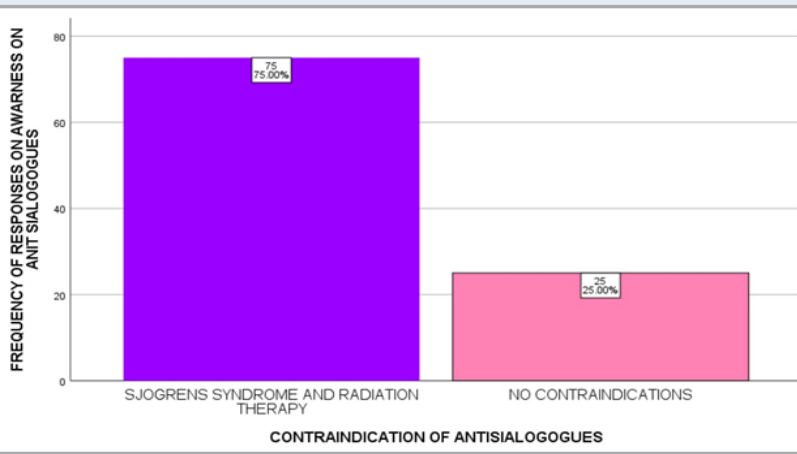

$75 \%$ of the study population answered correctly that anti sialogogues are contraindicated in patients with Sjögren's syndrome and radiation therapy (violet colour). 25\% of the study population thought there were no contradictions for the usage of anti sialogogues. (Pink colour).

The main limitations of the study is its limited sample size. Though all the students of the college when included in the study the sample size was limited. The study was confined to a single university and was restricted geographically. In the future, further studies CME and CDE programs can be conducted on antisialogogues, to increase the awareness and knowledge on antisialogogues. In doing so the quality and the efficiency of the treatment provided in the clinical setup can be improved upon. Furthermore, in the future randomised control trials can be conducted on patients with hypersalivation undergoing treatment with anti sialogogues and without anti sialogogues.

\section{CONCLUSION}

Anti- sialogogues are less commonly used in the day today dental practices as there are more non invasive, and topical methods of controlling hypersalivation in dental practice. But they are still used in severe cases of hypersalivation during surgeries. According to the study done the students had adequate knowledge about anti sialogogue drugs. The study says that it doesn't decrease the chair time, but it can be used in cases with hypersalivation. Further research must be done to find the efficacy of anti sialogogue and the knowledge, usage of anti sialogogues usage among the dental students can be improved by conducting various conferences and CME (continuing medical education) programmes.

\section{ACKNOWLEDGEMENTS}

This research was done under the supervision of the Department of Research of Saveetha Dental College and Hospitals. We sincerely show gratitude to the corresponding guide who provided insights and expertise that greatly assisted the research.

Conflict of Interest: None declared 


\section{REFERENCES}

Anbu, R. T. et al. (2019) 'Comparison of the Efficacy of Three Different Bone Regeneration Materials: An Animal Study', European journal of dentistry, 13(1), pp. 22-28.

Ariga, P. et al. (2018) 'Determination of Correlation of Width of Maxillary Anterior Teeth using Extraoral and Intraoral Factors in Indian Population: A Systematic Review', World Journal of Dentistry, 9(1), pp. 68-75.

Ashok, V. and Ganapathy, D. (2019) 'A geometrical method to classify face forms', Journal of oral biology and craniofacial research, 9(3), pp. 232-235.

Chainani-Wu, N. et al. (2006) 'Assessment of the use of sialogogues in the clinical management of patients with xerostomia', Special Care in Dentistry, pp. 164-170. doi: 10.1111/j.1754-4505.2006.tb01719.x.

Christensen, C. M. (1986) 'Importance of Saliva in Diet-Taste Relationships', Interaction of the Chemical Senses with Nutrition, pp. 3-24. doi: 10.1016/b978-012-397855-4.50008-6.

Duraisamy, R. et al. (2019) ‘Compatibility of Nonoriginal Abutments With Implants: Evaluation of Microgap at the Implant-Abutment Interface, With Original and Nonoriginal Abutments', Implant dentistry, 28(3), pp. 289-295.

Edwards, A. V. (1998) 'Autonomic Control of Salivary Blood Flow', Frontiers of Oral Biology (Vol. 10 11), pp. 101-117. doi: 10.1159/000061091.

'Evaluation of Corrosive Behavior of Four Nickelchromium Alloys in Artificial Saliva by Cyclic Polarization Test:An in vitro Study' (2017) World Journal of Dentistry, 8(6), pp. 477-482.

Ganapathy, D. M., Kannan, A. and Venugopalan, S. (2017) 'Effect of Coated Surfaces influencing Screw Loosening in Implants: A Systematic Review and Meta-analysis', World Journal of Dentistry, 8(6), pp. 496-502.

Gupta, P., Ariga, P. and Deogade, S. C. (2018) 'Effect of Monopoly-coating Agent on the Surface Roughness of a Tissue Conditioner Subjected to Cleansing and Disinfection: A Contact Profilometric Study', Contemporary clinical dentistry, 9(Suppl 1), pp. S122S126.

Gurney, B. F. (1967) 'Chemotherapy in dental practice. Antisialagogues', Dental digest, 73(6), pp. 270-271. Hypersalivation (Ptyalism)' (2012) Clinical Veterinary Advisor, p. 874. doi: 10.1016/b978-1-4160-9979-
6.00314-7.

Jain, A. R. (2017a) 'Clinical and Functional Outcomes of Implant Prostheses in Fibula Free Flaps', World Journal of Dentistry, 8(3), pp. 171-176.

Jain, A. R. (2017b) 'Prevalence of Partial Edentulousness and Treatment needs in Rural Population of South India', World Journal of Dentistry, 8(3), pp. 213-217.

Kuijpers, M. A. R. et al. (2010) 'The Effect of Antisialogogues in Dentistry', The Journal of the American Dental Association, pp. 954-965. doi: 10.14219/jada.archive.2010.0309.

Leone, J., Georgievski, Z. and Koklanis, K. (2010) 'Atropine Treatment of Amblyopia: Is a Swap in Fixation Necessary?', Journal of Pediatric Ophthalmology \& Strabismus, pp. 270-276. doi: 10.3928/0191391320091118-05.

Mccubbin, T. D. et al. (1979) 'GLYCOPYRROLATE AS A PREMEDICANT: COMPARISON WITH ATROPINE', British Journal of Anaesthesia, pp. 885-889. doi: 10.1093/bja/51.9.885.

Murai, S. et al. (1996) 'Effects of short-term (2 weeks) streptozotocin-induced diabetes on acetylcholine and noradrenaline in the salivary glands and secretory responses to cholinergic and adrenergic sialogogues in mice', Archives of Oral Biology, pp. 673-677. doi: 10.1016/s0003-9969(96)00042-8.

Ranganathan, H., Ganapathy, D. M. and Jain, A. R. (2017) 'Cervical and Incisal Marginal Discrepancy in Ceramic Laminate Veneering Materials: A SEM Analysis', Contemporary clinical dentistry, 8(2), pp. 272-278.

Revathy, G. (2017) 'KNOWLEDGE, ATTITUDE AND PRACTICE OF ANTI-SIALAGOGUE AMONG DENTAL STUDENTS', IJSDR, 2, pp. 302-305.

Saleh, A. and Elazzazi, H. (2014) 'Comparison between different atropine doses as an antisialagogue for patients receiving ketamine-midazolam undergoing gamma knife radiosurgery', Ain-Shams Journal of Anaesthesiology, p. 336. doi: 10.4103/1687-7934.139560.

Singh, H. et al. (2020) 'Efficacy of Botulinum Toxin for Treating Sialorrhea in Neuromuscular Conditions', Frontiers in neurology, 11, p. 513.

Varghese, S. S., Ramesh, A. and Veeraiyan, D. N. (2019) 'Blended Module-Based Teaching in Biostatistics and Research Methodology: A Retrospective Study with Postgraduate Dental Students', Journal of dental education, 83(4), pp. 445-450. 